\title{
ARTICLE \\ Succinate receptor mediates intestinal inflammation and fibrosis
}

Dulce C. Macias-Ceja ${ }^{1}$, Dolores Ortiz-Masiá ${ }^{2}$, Pedro Salvador ${ }^{3}$, Laura Gisbert-Ferrándiz ${ }^{3}$, Carlos Hernández ${ }^{1}$, Martin Hausmann ${ }^{4}$, Gerhard Rogler ${ }^{4}$, Juan V. Esplugues ${ }^{1,3}$, Joaquín Hinojosa ${ }^{5}$, Rafael Alós ${ }^{6}$, Francisco Navarro ${ }^{5}$, Jesus Cosin-Roger ${ }^{1}$, Sara Calatayud ${ }^{3}$ and María D. Barrachina ${ }^{3}$

Succinate, an intermediate of the tricarboxylic acid cycle, is accumulated in inflamed areas and its signaling through succinate receptor (SUCNR1) regulates immune function. We analyze SUCNR1 expression in the intestine of Crohn's disease patients and its role in murine intestinal inflammation and fibrosis. We show that both serum and intestinal succinate levels and SUCNR1 expression in intestinal surgical resections were higher in CD patients than in controls. SUCNR1 co-localized with CD86, CD206, and a-SMA+ cells in human intestine and we found a positive and significant correlation between SUCNR1 and a-SMA expression. In human isolated fibroblasts from CD patients SUCNR1 expression was higher than in those from controls and treatment with succinate increased SUCNR1 expression, fibrotic markers and inflammatory cytokines through SUCNR1. This receptor modulated the expression of pro-inflammatory cytokines in resting murine macrophages, macrophage polarization and fibroblast activation and Sucnr $1^{-1-}$ mice were protected against both acute TNBS-colitis and intestinal fibrosis induced by the heterotopic transplant of colonic tissue. We demonstrate increased succinate levels in serum and SUCNR1 expression in intestinal tissue of CD patients and show a role for SUCNR1 in murine intestinal inflammation and fibrosis.

Mucosal Immunology (2019) 12:178-187; https://doi.org/10.1038/s41385-018-0087-3

\section{INTRODUCTION}

Crohn's disease (CD) is a chronic inflammatory disorder of the gastrointestinal tract characterized by initial transmural inflammation that is commonly associated with complications, such as strictures, abscesses, and fistulas. ${ }^{1-3}$ Inflammation is necessary for fibrosis development but current anti-inflammatory therapy does not prevent fibrosis in susceptible patients, which in most cases leads to surgery. To improve the clinical management of the disease, new putative targets should be identified from a better knowledge of fibrosis etiopathogenesis.

The development of fibrosis is associated with chronic, recurrent or unresolved intestinal inflammation. ${ }^{4}$ At sites of inflammation, hypoxia, oxidative stress, and necrosis of inflamed tissue determine important metabolic changes that have been associated with an increase in the local concentration of succinate, an intermediate of the tricarboxylic acid cycle., ${ }^{5,6}$ Pro-inflammatory macrophages may be partly responsible for these metabolic alterations as macrophages stimulated with bacterial lipopolysaccharide (LPS) increase glycolysis and reduce TCA cycle activity and mitochondrial respiration, which provokes an accumulation of succinate. ${ }^{7}$ Interestingly, recent evidences suggest that similar metabolic changes may take place in fibroblast activation and fibrosis development. ${ }^{8-10}$

In last years, new roles for succinate beyond metabolism have emerged and it has been shown that accumulation of this metabolite can act as a signal for inflammation. ${ }^{5}$ In these conditions, succinate is released from the mitochondria into the extracellular environment where it selectively binds to the Gcoupled receptor 91, GPR91, also named succinate receptor (SUCNR1). ${ }^{11,12}$ This receptor is expressed in several cell types including immune cells ${ }^{13}$ and its activation by succinate induces pro-inflammatory signaling pathways. ${ }^{5}$ In this line, in human monocyte-derived dendritic cells, SUCNR1 stimulation by succinate triggers intracellular calcium mobilization and ERK1/ 2 phosphorylation ${ }^{14}$ and promotes cellular migration and the production of pro-inflammatory cytokines in macrophages. ${ }^{15,16}$ Of note, increased levels of succinate and the resultant autocrine and paracrine signaling through SUCNR1 have been recently shown to exacerbate and sustain inflammation in chronic pathological conditions such as rheumatoid arthritis ${ }^{15}$ or obesity. ${ }^{17}$

In the present study, we aimed to determine whether succinate levels and the intestinal expression of SUCNR1 are modified by Crohn's disease and to analyze the possible role of this receptor in murine models of intestinal inflammation and fibrosis. Our results, by showing the enhanced SUCNR1 expression in intestinal tissue from $C D$ patients and the involvement of this receptor in murine colitis and fibrosis, lead us to identify SUCNR1 as a potential target for Crohn's disease.

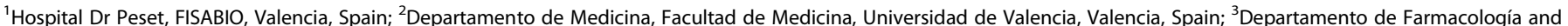

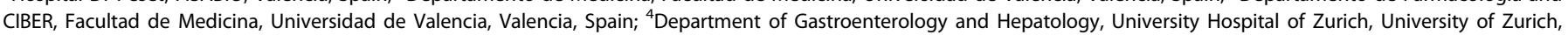
Zurich, Switzerland; ${ }^{5}$ Hospital de Manises, Valencia, Spain and ${ }^{6}$ Hospital De Sagunto, Valencia, Spain

Correspondence: Jesus Cosin-Roger (jesus.cosin@uv.es)

These authors contributed equally: Dulce C. Macias-Ceja, Dolores Ortiz-Masiá, Sara Calatayud, María D. Barrachina.

Received: 26 April 2018 Revised: 2 August 2018 Accepted: 1 September 2018

Published online: 2 October 2018 


\section{RESULTS}

Serum and intestinal succinate levels and SUCNR1 intestinal expression are increased in CD patients

Serum succinate levels were significantly higher in samples from CD patients than in serum from healthy donors (Fig. 1a). In parallel, succinate levels in intestinal mucosa from $C D$ patients were significantly higher than the ones from non-IBD patients (Fig. 1b). Increased SUCNR1 mRNA expression and enhanced SUCNR1 protein levels were detected in intestinal tissue from $C D$ patients compared with those detected in samples from non-IBD patients (Fig. 1c). SUCNR1 staining was detected in epithelial cells and cells present in the lamina propria of human intestine (Fig. 1d). Immunofluorescence experiments confirmed the expression of SUCNR1 in the cellular membrane and revealed that SUCNR1 colocalized with CD206, CD86, and a-SMA positive cells in this tissue (Fig. 1e).

SUCNR1 correlates with markers of fibrosis in intestinal tissue and it is increased in fibroblasts from $C D$ patients

The mRNA expression of a-SMA was significantly higher in intestinal tissue from $C D$ patients than in that from control patients (Fig. 2a) and a positive and significant correlation was detected between levels the of SUCNR1 and a-SMA mRNA in intestinal tissue (Fig. 2b) suggesting a role for this receptor in human intestinal fibrosis.

An increased expression of SUCNR1 was detected in primary fibroblasts isolated from intestinal tissue of $C D$ patients compared with those obtained from non-IBD patients (Fig. 2c). Treatment of fibroblasts with different concentrations of succinate showed a dose-dependent increase in the mRNA expression of SUCNR1, markers of fibrosis (COL1a1, a-SMA), the pro-fibrotic factor TGF $\beta$ and the pro-inflammatory cytokine IL1 $\beta$ (Fig. $2 d$ ). Succinate also increased, in a dose-dependent manner, the protein levels of SUCNR1 and ASMA and the phosphorylation of a pro-fibrotic transcription factor (STAT3) and a pro-inflammatory transcription factor (NF-KB) (Fig. 2e). Neither induction of SUCNR1 nor phosphorylation of STAT3 or NF-KB was observed in fibroblasts treated with TGF $\beta$. The specific activation of SUCNR1 receptor by succinate was confirmed by measuring levels of intracellular calcium. Succinate induces a significant increase in the amount of intracellular calcium in a dose-response manner whereas TGF- $\beta$ failed to do that (Fig. 2f).

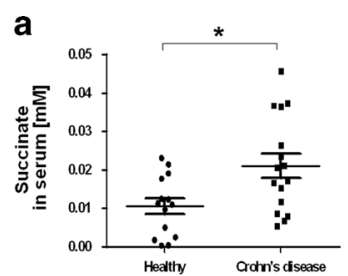

C
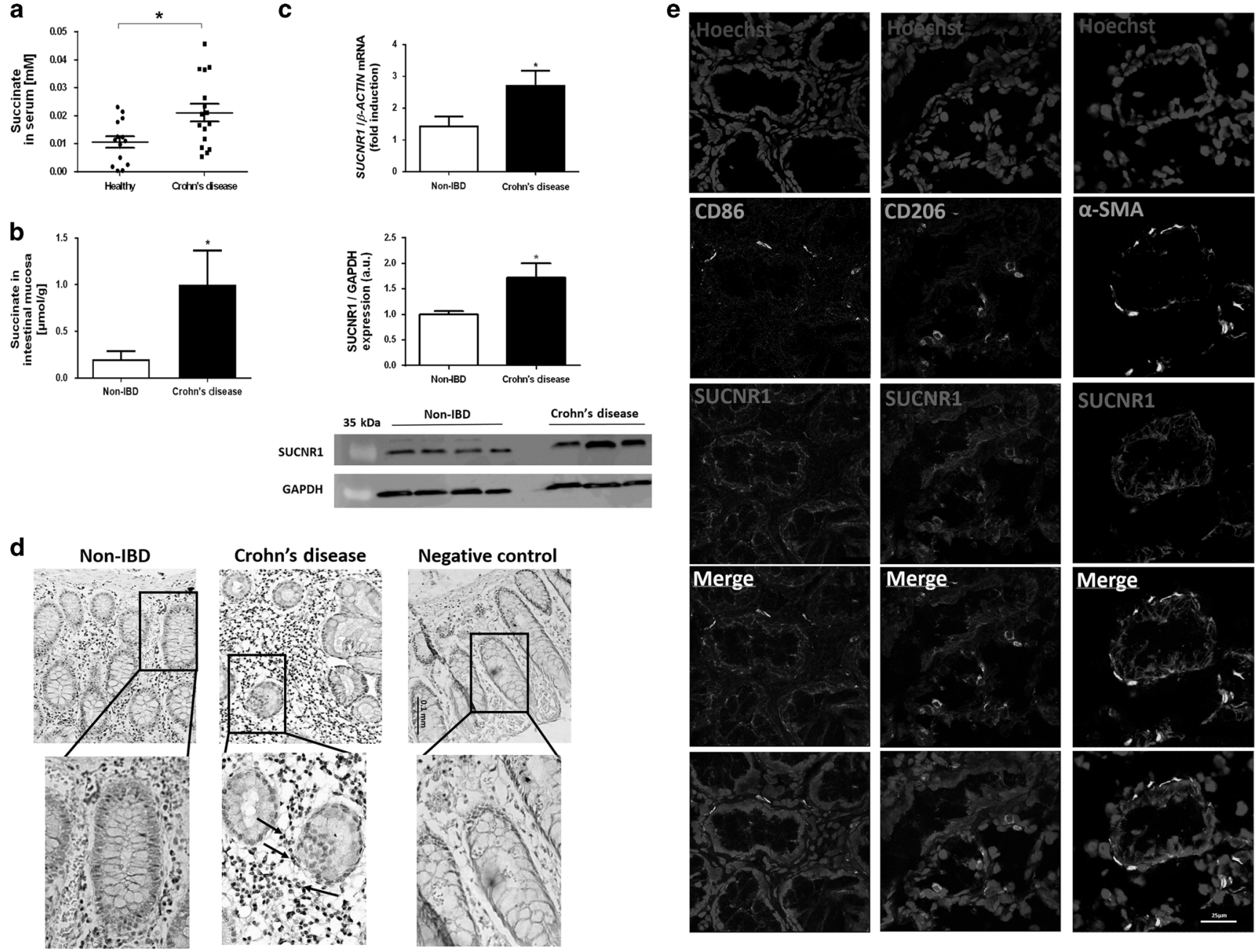

Fig. 1 Serum and intestinal succinate levels and SUCNR1 intestinal expression are increased in CD patients. a Graph shows succinate levels in serum from healthy donors $(n=14)$ and CD patients $(n=16)$. $\mathbf{b}$ Graph shows succinate levels from intestinal mucosa from non-IBD $(n=5)$ and CD patients $(n=5)$. c Graphs show, in intestinal resections, Sucnr1mRNA expression in CD patients $(n=40)$, expressed as fold induction vs non-IBD $(n=40)$ and SUCNR1 protein levels in non-IBD $(n=5)$ and CD patients $(n=6)$. A representative western blot showing SUCNR1 protein levels in intestine from control and CD patients. $\mathbf{d} A$ representative image showing SUCNR1 immunostaining in human intestine from non-IBD patients, of a total of five per group. e A representative immunofluorescence images taken with the confocal microscope Leica TCS SP8 showing co-localization of SUCNR1 in CD86, CD206, and $\alpha$-SMA positive cells in the lamina propria of human intestine, of a total of five per group. Bars in graphs represent mean \pm s.e.m. and significant differences vs the control group are shown by $* P<0.05$ 
a

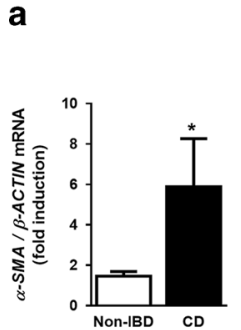

b
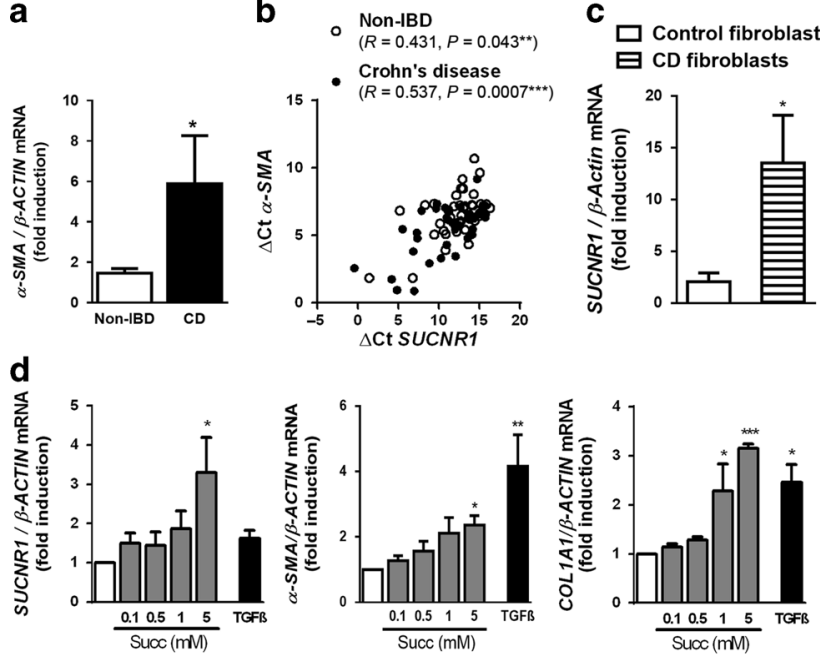

C $\square$ Control fibroblasts CD fibroblasts

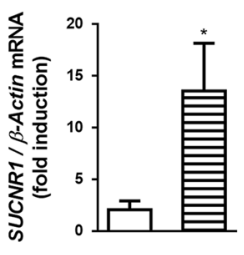

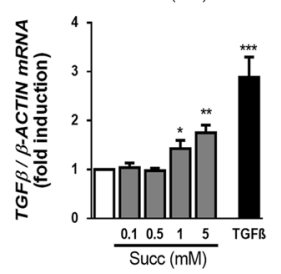

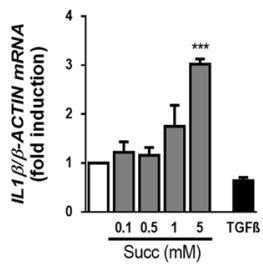

e
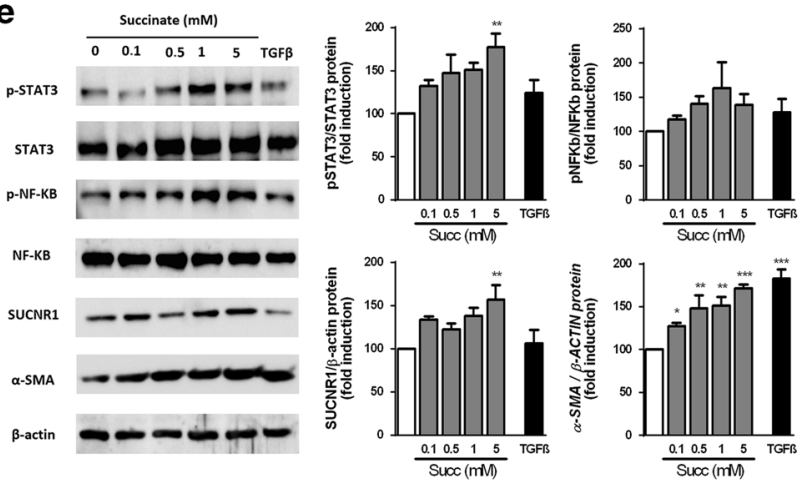

f

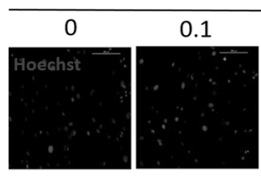

Succinate $(\mathrm{mM})$
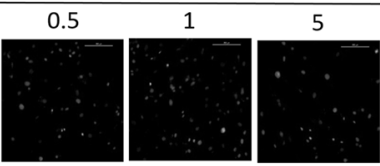

TGF- $\beta$
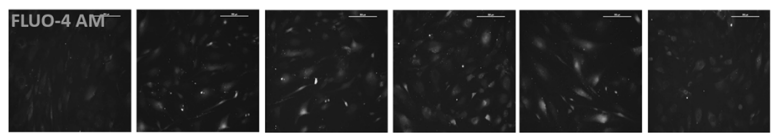

g
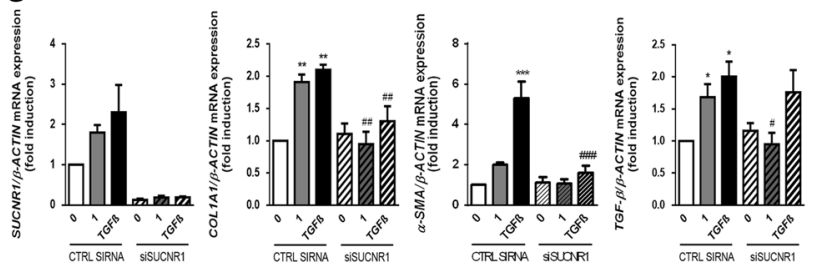

To confirm that SUCNR1 mediates the effects induced by succinate, we knocked down this receptor in primary fibroblasts. The efficiency of the transfection reached an $80 \%$ reduction in the expression of SUCNR1 receptor (Fig. 2g). Succinate treatment
Fig. 2 Increased SUCNR1 expression in human intestinal fibroblasts isolated from CD. a mRNA expression of aSMA in intestinal tissue from non-IBD $(n=40)$ and CD patients $(n=40)$. Bars in graph represent mean \pm s.e.m and significant differences from non-IBD patients is shown by ${ }^{*} P<0.05$. b $A$ positive and significant correlation is detected between the expression of SUCNR1 and $a$ $S M A$ in human intestinal tissue. c Primary fibroblasts were isolated from the intestine of non-IBD $(n=6)$ and CD patients $(n=6)$ and graph shows SUCNR1 mRNA expression (expressed as fold induction); significant differences from control fibroblasts is shown by ${ }^{*} P$ $<0.05$. d Fibroblasts were treated with different concentrations of succinate $(0,0.1,0.5,1$, and $5 \mathrm{mM})$ or TGF $\beta(5 \mathrm{ng} / \mathrm{ml})$ for $24 \mathrm{~h}$. Graphs show the effects of succinate and TGF $\beta$ on the mRNA expression of SUCNR1, COL1A1, $\alpha S M A, I L 1 \beta$, and TGF $\beta$. e Graphs and representative western blots of a total of four showing the effects of different concentrations of succinate $(0,0.1,0.5,1$, and $5 \mathrm{mM})$ or TGF $\beta$ on

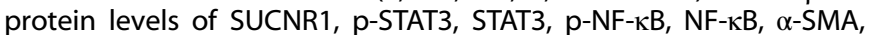
and $\beta$-actin. Bars in graphs represent mean \pm s.e.m. Significant differences from vehicle-treated fibroblasts is shown by ${ }^{*} P<0.05$, ${ }^{*} P<0.01$, and ${ }^{* *} P<0.001$. f Intracellular calcium was measured using the Fluo-4 AM dye. Representative pictures of one out of three independent experiments. g Primary fibroblasts were transfected with specific SUCNR1 siRNA and treated with succinate $1 \mathrm{mM}$ of TGF- $\beta 5 \mathrm{ng} / \mathrm{ml}$ for $24 \mathrm{~h}$. Graphs show the SUCNR1, COL1A1, $\alpha-S M A$, and TGF- $\beta$ mRNA expression (expressed as fold induction). Significant differences $v s$ non treated cells are shown by $* P<0.05$, ${ }^{*} P<0.01$, and ${ }^{* * *} P<0.001$ and significant differences vs respective control siRNA cells are shown by $\# P<0.05, \# \# P<0.01$, and \#\#\#P< 0.001

failed to induce the expression of COL1A1, $\alpha-S M A$, nor TGF- $\beta$ when SUCNR1 was knocked down. Of interest, TGF- $\beta$ treatment neither induced the expression of COL1A1, $a-S M A$, nor TGF- $\beta$ in the absence of SUCNR1 receptor (Fig. 2g).

\section{SUCNR1-deficient mice are protected from colitis}

We used an experimental mouse model of inflammatory bowel disease based on the intrarectal administration of TNBS to mice. This sensitizing agent induced, 2 days after its administration, a loss-of-body weight, a diminution in colon length and an increase of the histological damage score due to the inflammatory infiltrate and the slight disruption of the epithelial architecture (Fig. 3a-c). A significant increased mRNA expression of Sucnr1 was detected in the colon of TNBS-treated mice compared with levels detected in vehicle-treated mice (Fig. 3d). Administration of TNBS to Sucnr1 ${ }^{-1}$

mice showed that the loss-of-body weight was significantly attenuated compared with the one detected in TNBS-treated WT mice and the histological damage and the reduction in colon length were significantly prevented (Fig. 3a-c).

Two days after TNBS administration, a significant increase in the expression of M1 markers (Cd11c and iNOS), and pro-inflammatory cytokines (II1 $\beta, I / 6$, and Tnfa,) was detected in colonic tissue from WT mice compared with vehicle-treated mice while the expression of M2 markers (Cd206, Fizz1, Ym1, and Argl) was not significantly modified (Fig. 3e). In Sucnr $1^{-1-}$ mice, TNBS failed to significantly modify the expression of M1 markers, pro-inflammatory cytokines and M2 markers with the exception of Fizz1 that it was significantly reduced (Fig. 3e). A detailed analysis between TNBS-treated WT and Sucnr1 $1^{-1-}$ mice revealed a different expression of Cd11c, iNOS, Fizz1, Tnfa, II1 $\beta, 1 / 6$, and II10 (Fig. 3e). In vehicle-treated Sucnr1 ${ }^{-1-}$ mice, higher mRNA levels of the M2 markers were detected compared with vehicle-treated WT mice, which reached statistical significance for Cd206 and Fizz1 (Fig. 3e).

SUCNR1 mediates the expression of pro-inflammatory cytokines in macrophages

Peritoneal macrophages were isolated from both WT and Sucnr1 $1^{-1-}$ mice and data showed that the expression of pro-inflammatory cytokines (II1 $\beta, \| 6$, and TNFa) was significantly lower in macrophages 
a

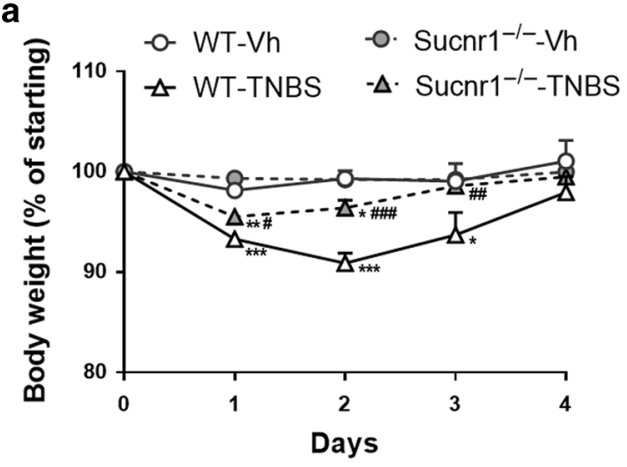

C WT Vh

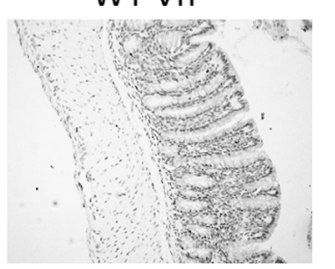

Sucnr1-/- Vh

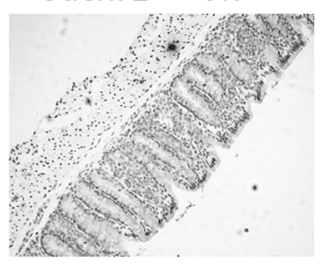

e
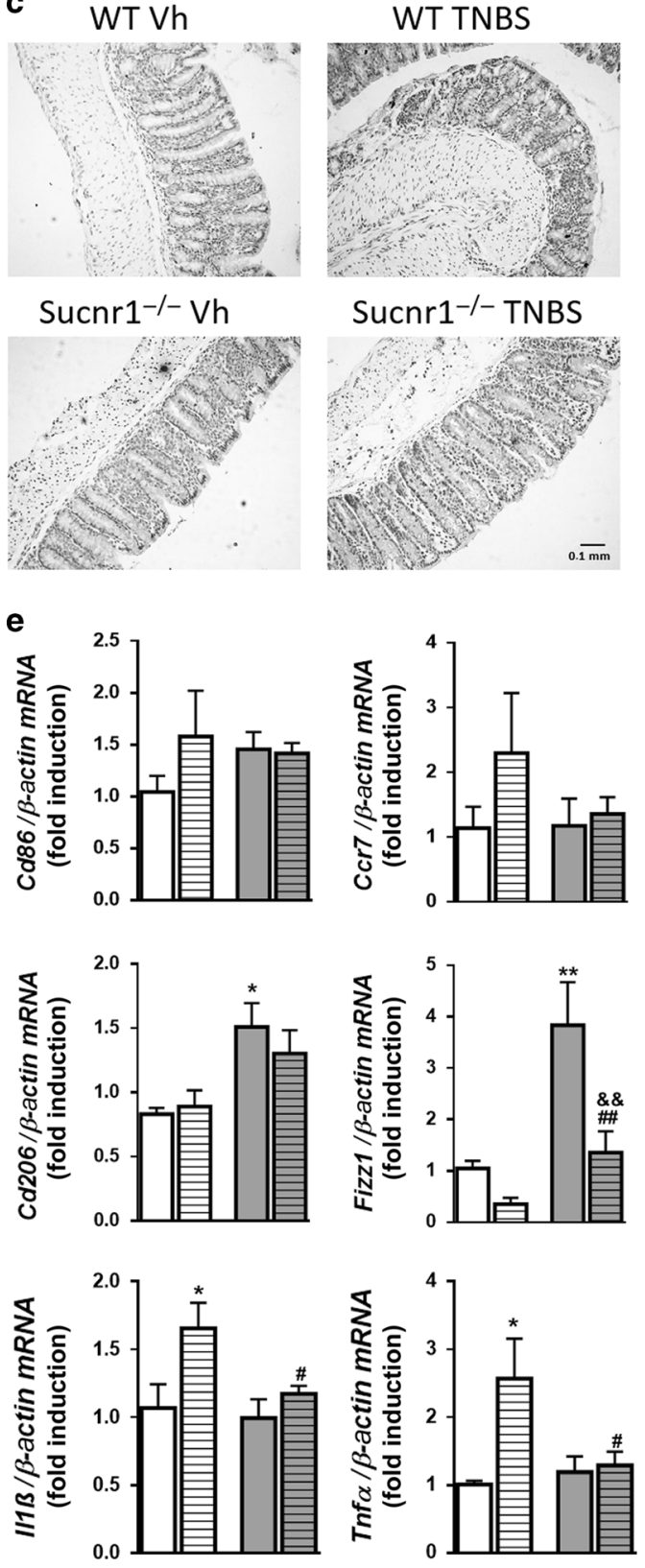

b

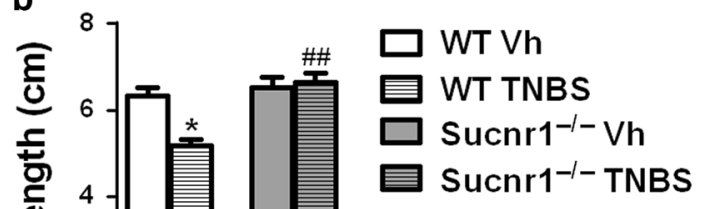

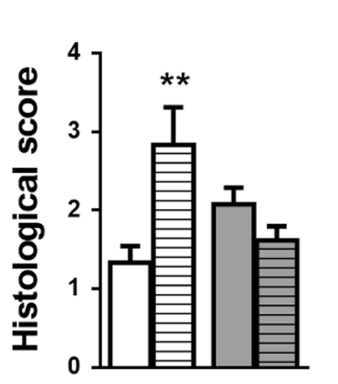
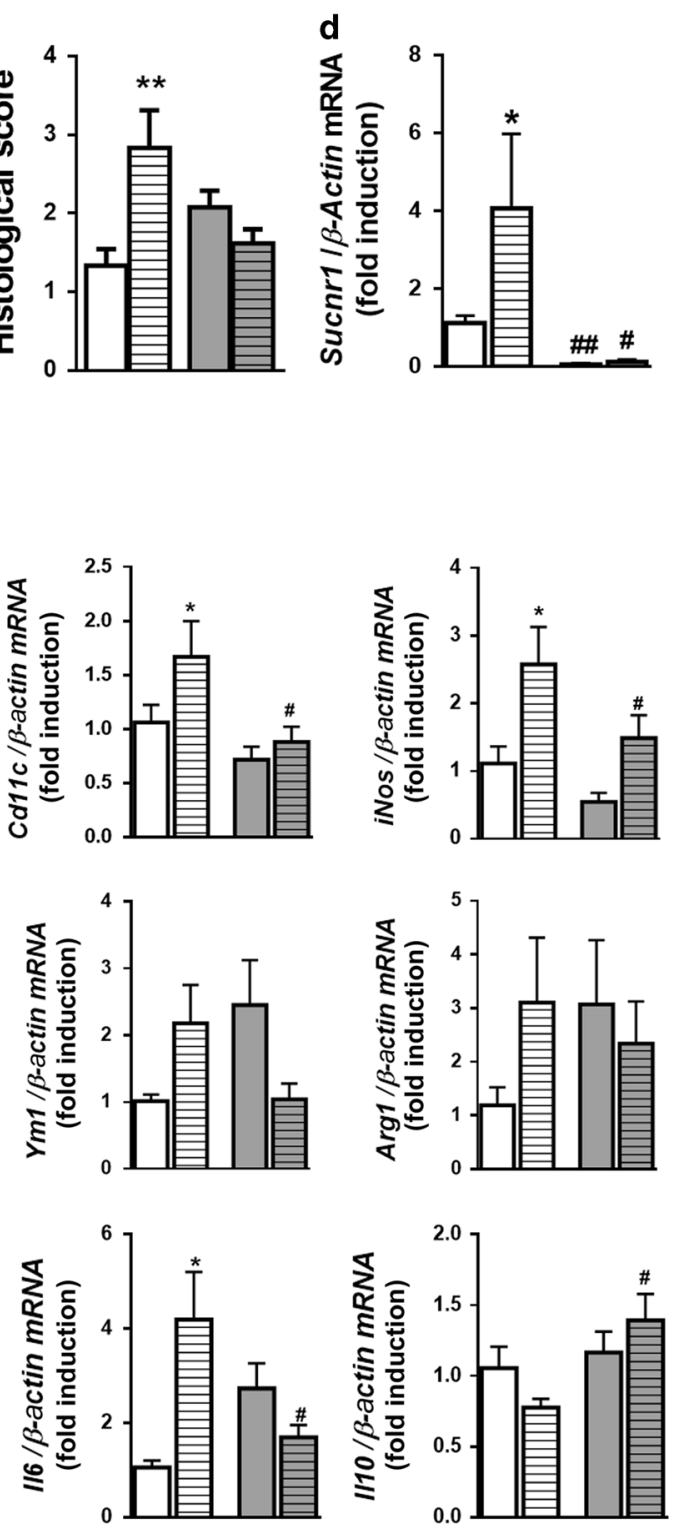

isolated from Sucnr1 $1^{-1-}$ mice than in those derived from WT mice while no significant changes were detected in the expression of Cd86, Cd206, and I/10 (Fig. 4). After treatment with LPS + IFNy, macrophages from Sucnr1 $1^{-/-}$animals exhibited a significant

diminution in $I 11 \beta, \| 6$, and $C d 206$ and increased levels of Cd86 compared with the ones detected in macrophages from WT mice (Fig. 4). M2a polarization by treatment of macrophages with IL4 revealed that, macrophages from Sucnr $1^{-1-}$ mice exhibited 
Fig. 3 SUCNR1-deficient mice are protected from colitis. Acute colitis was induced in both wild type (WT, $n=23)$ and Sucnr $1^{-1-}$ mice $(n=25)$ by intrarectal administration of TNBS (3.5 mg per $20 \mathrm{~g}$ mice) or vehicle (EtOH 40\%, Vh). Mice were killed 2 days after TNBS-administration. Graphs show: a body weight as a percentage of starting weight, measured daily; $\mathbf{b}$ colon length; $\mathbf{c}$ histological damage score (according to the Wallace score parameters) and representative photographs of the mucosa; d Sucnr1 mRNA expression in intestinal tissue (expressed as fold induction); e Relative mRNA expression levels of M1 markers (Cd86, CcrR7, Cd11c, and iNOS), M2 markers (Cd206, Fizz1, Ym1, and Arg1) and proinflammatory (Tnfa, II-6 and II-1 $\beta$ ) and anti-inflammatory cytokines (II-10) in both vehicle- and damaged-colon of TNBS-treated mice vs the housekeeping gene $\beta$-actin and represented as fold induction vs vehicle WT group. Points or bars in graphs represent mean \pm s.e.m. Significant differences in relation to the WT vehicle-treated group are shown by ${ }^{*} P<0.05$ and ${ }^{* *} P<0.01$; from the WT TNBS-treated mice by $\# P<0.05$ and $\# \# P<0.01$ and from the Sucnr $1^{-1-}$ vehicle-treated group by $\& \& P<0.01$
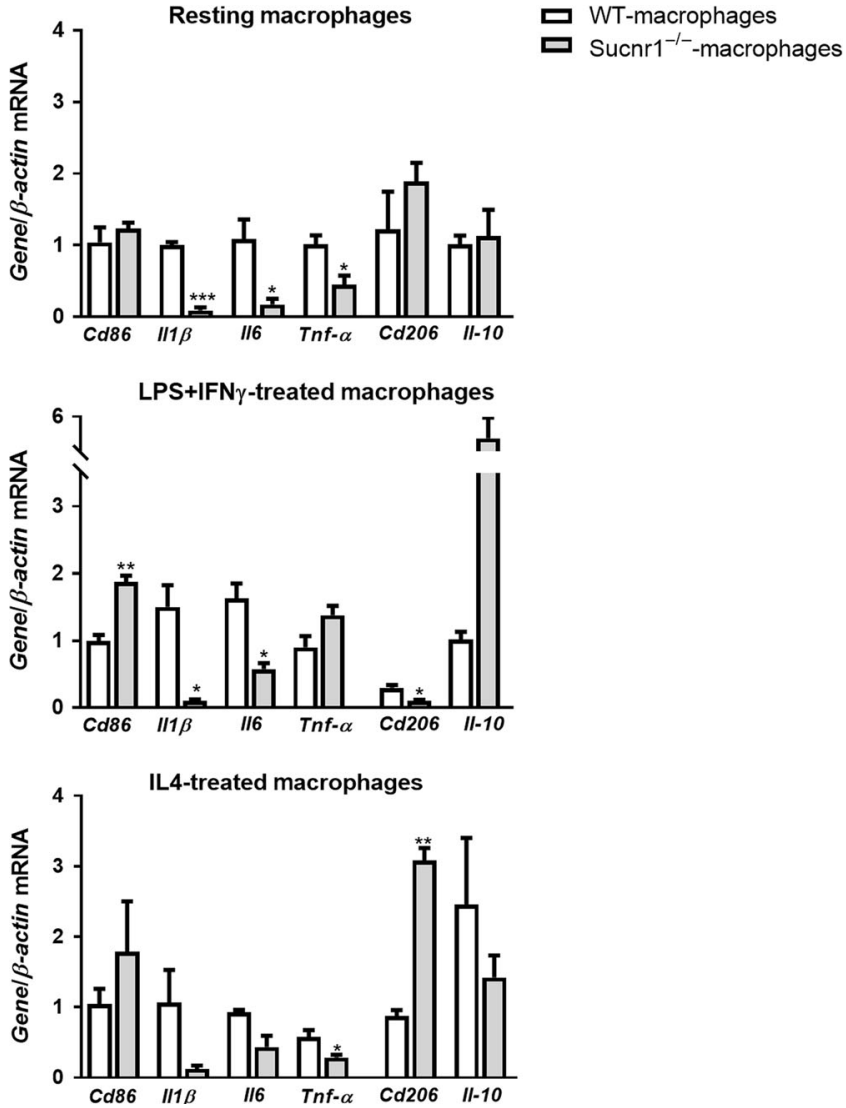

Fig. 4 SUCNR1 mediates the expression of pro-inflammatory cytokines in macrophages. Murine peritoneal macrophages were obtained from WT $(n=3)$ and Sucnr1 $1^{-1-}$ mice $(n=3)$ and polarized towards M1 or M2a macrophages with lipopolysaccharide and interferon- $\gamma$ (LPS and IFN- $\gamma$ ) for $24 \mathrm{~h}$ or interleukin-4 (IL4) for $72 \mathrm{~h}$, respectively. Graphs show gene/ $\beta$-actin mRNA expression (fold induction vs resting WT macrophages). Significant differences from the respective group of WT macrophages are shown by ${ }^{*} P<0.05$, ${ }^{* *} P<0.01$, and ${ }^{* * *} P<0.001$

significant lower levels of Tnfa and higher levels of Cd206, compared with the expression detected in WT macrophages (Fig. 4)

Lack of SUCNR1 protects against intestinal fibrosis

We have analyzed the role of SUCNR1 in the heterotopic transplant mouse model of intestinal fibrosis. Intestinal grafts from both WT and Sucnr $1^{-/-}$mice were explanted 7 days after transplantation, and fibrosis was detected by analyzing the Sirius Red staining under transmission and polarized light. As shown in Fig. 5a, explants from WT mice exhibited an increased collagen deposition compared with the thin collagen layer adjacent to the submucosa observed in intestinal tissue at day 0 . These explants also presented an increased expression of fibrotic markers ( $a-S M A$, Col1a1, and Vimentin), fibrogenic mediators (TGF $\beta$ ) and enhanced
Sucnr1 mRNA expression compared with that detected in the corresponding non-transplanted tissues (Fig. 5b, c). In contrast, grafts from Sucnr $1^{-/-}$mice exhibited similar collagen deposition (Fig. 5a) and similar mRNA levels of fibrotic markers between day 0 and day 7 , which in turn were similar to those detected at day 0 in tissues from WT mice (Fig. 5c). Of interest, grafts from Sucnr $1^{-1-}$ mice exhibited an increased mRNA expression of TGF $\beta 7$ days after transplantation that was similar to that detected in grafts from WT mice (Fig. 5c).

This model of intestinal fibrosis is characterized by macrophage infiltration from the host into the intestinal graft as revealed by the significant increase in the mRNA expression of $F 4 / 80$ observed (Fig. 5e) and by the fact that Sucnr1 expression was detected in grafts from Sucnr $1^{-1-}$ mice, 7 days after transplantation (Fig. 5b). The increase in the $F 4 / 80$ expression was similar in explants from WT and Sucnr1 ${ }^{-1-}$ mice but the mRNA expression of proinflammatory agents and macrophage markers significantly differed. An important increase in $\| 1 \beta$, iNOS, $116, C d 86$, and $C d 16$ was detected in intestinal tissue from WT mice, 7 days after transplantation (Fig. 5 d, e); increased levels of $I / 1 \beta$, iNOS, and Cd86 were also observed in grafts from Sucnr1 $1^{-1-}$ mice but they were significantly lower than those detected in WT grafts (Fig. 5d, e). In contrast, the expression of $116,1 / 10, C d 206$, and Cd16 was significantly higher in explanted tissue from Sucnr $1^{-1-}$ mice than in WT grafts.

Finally, primary fibroblasts were isolated from both WT and Sucnr $1^{-1-}$ colonic tissues and were treated with succinate $(1 \mathrm{mM})$ or TGF $\beta(5 \mathrm{ng} / \mathrm{ml})$. As shown in Fig. $5 \mathrm{f}$, succinate but not TGF $\beta$ induced a significant increase in the mRNA expression of Sucnr1. However, both treatments significantly enhanced the expression of Col1a1 in WT fibroblasts and not in Sucnr $1^{-/-}$cells, showing a role for Sucnr1 in the activation of fibroblasts induced by both succinate and TGF $\beta$ in mice (Fig. $5 f$ ).

\section{DISCUSSION}

The present study shows increased serum and intestinal levels of succinate and enhanced intestinal expression of SUCNR1 in CD patients. This receptor exacerbates the pro-inflammatory activity of macrophages and fibroblast activation and plays a role in murine colitis and intestinal fibrosis.

SUCNR1 belongs to the family of the metabolite-sensing G-protein-coupled receptors and it binds selectively to succinate. The activation of SUCNR1 by succinate has been revealed as important in the regulation of immune and metabolic functions. ${ }^{2}$ The present study localizes SUCNR1 in epithelial cells, macrophages, and fibroblasts of human intestine, and shows for the first time an increased expression of SUCNR1 in intestinal tissue from $C D$ patients compared with the one detected in non-IBD subjects. Previous studies have reported the upregulation of SUCNR1 by succinate ${ }^{8,15,18}$ and our data reveal increased levels of succinate in both the damaged intestinal tissue and serum from CD patients compared with those detected in controls. It seems likely that succinate accumulates in cells of the damaged intestine where the described stimuli for its release-hypoxia, ${ }^{16,19}$ high proinflammatory activity of macrophages ${ }^{7,15}$ and necrosis in 
a
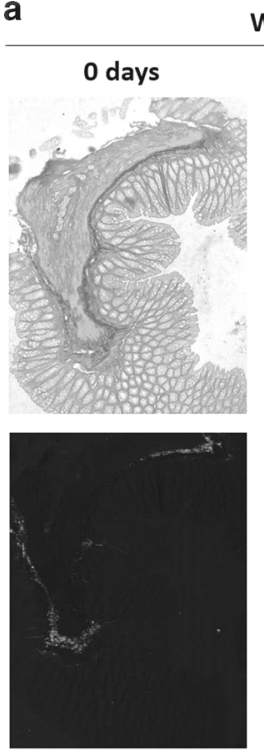

WT
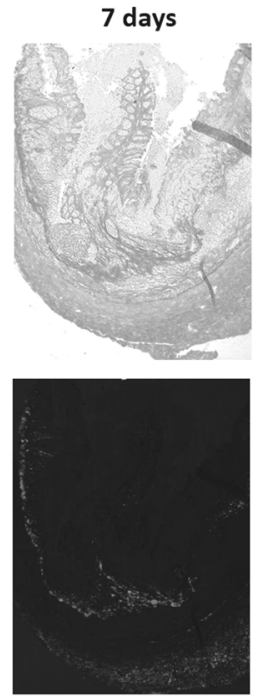

b

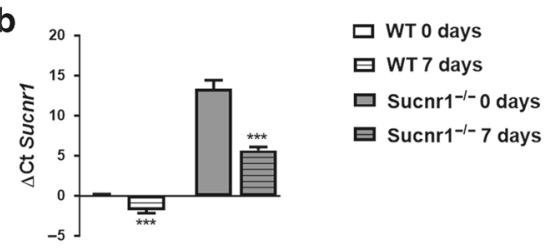

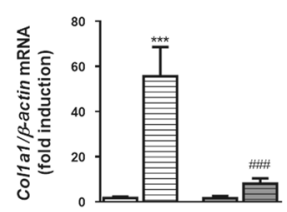
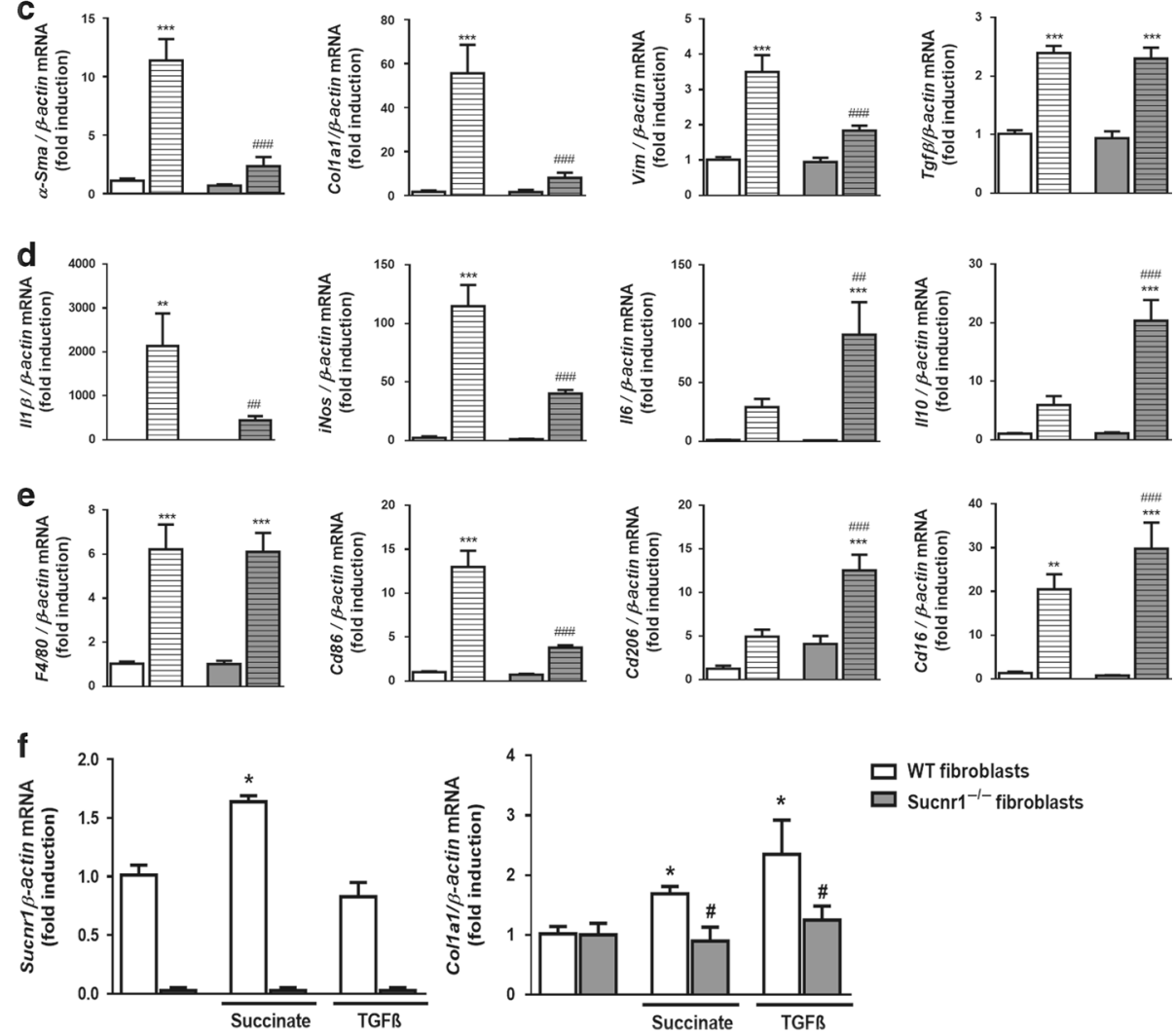

inflamed tissue ${ }^{14}$-are present. From there, and once released to the extracellular milieu, succinate would reach the circulating blood. In this line, recent studies report increased levels of succinate in serum ${ }^{20}$ and feces ${ }^{21}$ in a murine model of colitis.
CD patients included in the present study exhibit a complicated behavior characterized by the presence of intestinal fibrosis. Our results demonstrate for the first time a higher expression of Sucnr1 in fibroblasts isolated from the intestine of $C D$ patients 
Fig. 5 SUCNR1-deficient mice are protected against intestinal fibrosis. Murine intestinal fibrosis was induced with a heterotopic intestinal transplant model. Intestinal grafts from both WT $(n=7)$ and Sucnr1 ${ }^{-1-}$ mice $(n=7)$ were obtained 7 days after transplantation. a Sirius Red staining was performed in paraffin-embedded tissues. Representative pictures taken under transmission and polarized light. b RNA from intestinal grafts from WT and Sucnr $1^{-1-}$ mice were isolated and the expression of Sucnr1 was analysed by qPCR. Graph shows the $\Delta$ Ct of each group. Significant differences from their respective intestinal graft at day 0 are shown by ${ }^{* *} P<0.001$. c-e Graphs show the expression of profibrotic markers (Col1a1, $\alpha$-SMA, Vimentin, and Tgf- $\beta$ ), pro-inflammatory cytokines (II1 $\beta$, iNos, and II-6), anti-inflammatory cytokine (II10) and macrophage markers (F4/80, Cd86, Cd206, and Cd16). Results were normalized with $\beta$-actin and are represented as fold induction vs intestinal grafts from WT at day 0 . Bars in graphs represent mean \pm s.e.m. Significant differences from intestinal WT grafts at day 0 is shown by $* * P<0.01$ or ${ }^{* * *} P<0.001$ and from intestinal WT grafts at day 7 by \#\#P<0.01 or \#\#\#P<0.001. f Primary murine intestinal fibroblasts were isolated from the colon of both WT $(n=4)$ and Sucnr $1^{-l-}$ mice $(n=4)$ and were treated with succinate $(1 \mathrm{mM})$ or TGF $\beta(5 \mathrm{ng} / \mathrm{ml})$ for $24 \mathrm{~h}$ and the mRNA expression of Sucnr1 and Col1a1 was analyzed by qPCR $(n=4)$. Bars in graphs represent mean \pm s.e.m. Significant differences from vehicletreated fibroblasts is shown by ${ }^{*} P<0.05$ and from the respective WT fibroblasts by $\# P<0.05$

than in those obtained from control patients. Exogenous succinate increased the mRNA and protein expression of this receptor in intestinal fibroblasts and activates pro-inflammatory and profibrotic pathways and mediators, which is in line with previous studies performed in hepatic stellate cells., ${ }^{8,18}$ Of interest, a dosedependent increase in calcium levels was detected in fibroblasts treated with succinate which, joined to results showing a reduced expression of pro-fibrotic markers in cells transiently transfected with siSUCNR1, demonstrate the role of the receptor in the effects of the metabolite These effects strongly suggest a role for sucnr 1 in intestinal fibrosis associated to $C D$, which is reinforced by the positive and significant correlation between SUCNR1 expression and aSMA detected in human intestine.

In Crohn's disease, the development of fibrosis is associated with chronic intestinal inflammation and we analyzed further the role of Sucnr 1 in intestinal inflammation and fibrosis by using Sucnr $1^{-1-}$ mice. We observed that the colitis induced by intrarectal administration of TNBS reproduces the increased Sucnr 1 expression detected in human samples from CD. In addition, and by using Sucnr1 $1^{-1-}$ mice, we demonstrated the involvement of this receptor in the body weight loss, the reduction in colon length and the intestinal damage induced by this haptenizing agent. This model of colitis is characterized by macrophage infiltration and increased expression of proinflammatory cytokines ${ }^{22,23}$ and these changes were significantly prevented in TNBS-treated Sucnr1 $1^{-/-}$mice. These observations joined to results obtained in isolated macrophages showing that, the absence of SUCNR1 significantly impairs the expression of proinflammatory cytokines in both constitutive and stimulated conditions, strongly support that Sucnr1 is modulating macrophage activation in vivo. Our data are in line with previous evidences ${ }^{15}$ and go further by showing that this receptor impairs the induced expression of M2 macrophage markers and antiinflammatory cytokines which suggest that Sucnr1 modulates macrophage polarization. In this line we found a diminished expression of M1 markers together with increased levels of $M 2$ markers in the intestine of vehicle- and TNBS-treated Sucnr1 ${ }^{-1-}$ mice.

Intestinal fibrosis associated to Crohn's disease is characterized by an intense submucosal and subserosal ECM deposition. ${ }^{2,24}$ Several animal models of intestinal fibrosis have been proposed ${ }^{25}$ and we consider that the heterotopic transplant model of fibrosis used in the present study reproduce some features of CD fibrosis, such as transmural inflammation and ECM deposition. ${ }^{26,27}$ We observed an important submucosal and subserosal collagen accumulation and high levels of fibrosis markers associated with an increased expression of SUCNR1 in WT grafts 7 days after transplantation. This receptor plays a fundamental role in intestinal fibrosis since little collagen deposition and low expression of fibrosis markers were detected in grafts from Sucnr1 ${ }^{-1-}$ mice. Increased F4/80 mRNA expression was detected in intestinal tissues from both Sucnr1 $1^{-1-}$ and WT mice, which shows that the absence of the receptor in the implants does not affect the ability of macrophages from the host to infiltrate the tissue. However, the pattern of gene expression of these macrophages significantly differed. Macrophage markers of a pro-inflammatory phenotype were highly expressed in WT grafts and much less in grafts from Sucnr $1^{-1}$ mice, that in turn, showed a higher expression of CD206, a marker of a regulatory macrophage phenotype. It seems likely that these differences, rather than cause, are consequence of the different extent of fibrosis, which in turn depends on the presence of sucnr 1 in those cells of the transplanted tissue mediating the fibrotic response. Of interest, graphs from both groups showed an increased expression of CD16, a recently reported marker of pro-fibrotic macrophages, $^{28}$ and of the well-known fibrogenic mediator, TGF $\beta .^{1}$ The presence of similar levels of these pro-fibrotic elements in both, WT and Sucnr $1^{-1-}$ mice suggest that fibrosis, in the heterotopic transplant model, may depend mainly on the pro-inflammatory cytokines, although, a reduced cellular reactivity to fibrogenesis resulting from the lack of Sucnr 1 in intestinal tissue cannot be ruled out. In this line, the development of fibrosis is largely dependent on fibroblasts activated by signals derived from immune and non-immune cells. ${ }^{29}$ Our results reproduce in murine intestinal fibroblasts the effects of succinate in human fibroblasts and go further by demonstrating that this metabolite stimulates these cells through Sucnr1 activation. Interestingly, we found that the absence of this receptor reduced the pro-fibrotic effects of TGF $\beta$ in these cells. A recent study shows increased levels of succinate in fibroblasts treated with $\mathrm{TGF} \beta{ }^{30}$ but our results showing no increase in calcium levels after treatment of fibroblasts with TGF $\beta$ makes this possibility unlikely. We propose that Sucnr1 exacerbates the response of fibroblasts to pro-fibrotic stimuli as it promotes the response of immune cells to proinflammatory agents. ${ }^{14,15}$ As a whole, our data suggest that the absence of Sucnr1 in fibroblasts determines to a large extent the reduced fibrotic response detected in intestinal tissue from Sucnr $1^{-1-}$ mice. Further studies using conditional deletion of Sucnr1 will help to clarify the specific cellular role that this receptor plays in intestinal fibrosis.

In summary, our results show that $C D$ patients present high levels of serum succinate and increased SUCNR1 expression in both surgical resections and intestinal fibroblasts and demonstrate for the first time a role for this receptor in murine intestinal inflammation and fibrosis. We propose that, in the intestinal microenvironment of $C D$ patients with a stricturing or penetrating behavior, SUCNR1 signaling exacerbates inflammation and fibroblasts activation, and point to this receptor as a possible new pharmacological target for Crohn's disease treatment.

\section{MATERIAL AND METHODS}

Patients

Blood and surgical resections from the damaged intestine were obtained from $C D$ patients with severe refractory $C D$ who had lost response to second line treatment, presenting a stricturing behavior (Montreal classification: B2) or a penetrating behavior (Montreal classification: B3) and requiring surgery (Table 1). Blood 
Table 1. Patient characteristics

\begin{tabular}{llll}
\hline & Non-IBD & $\begin{array}{l}\text { CD B2 } \\
\text { (structuring) }\end{array}$ & $\begin{array}{l}\text { CD B3 } \\
\text { (penetrating) }\end{array}$ \\
\hline $\begin{array}{l}\text { Number of patients } \\
\text { Age }(y)\end{array}$ & 40 & 25 & 15 \\
$15-40$ years & 22 & 13 & 9 \\
$>40$ years & 18 & 12 & 6 \\
$\begin{array}{l}\text { Gender } \\
\text { Male }\end{array}$ & 13 & 8 & \\
Female & 27 & 17 & 10 \\
Intestinal surgery & 40 & 25 & 15 \\
& & & \\
\hline
\end{tabular}

from healthy donors and surgical resections from the nondamaged intestine of patients with colorectal cancer (named as non-IBD patients) were used as controls (Table 1). The study was approved by the Institutional Review Board of both the Hospital of Manises (Valencia, Spain) and Hospital of Sagunto (Valencia, Spain). Written informed consent was obtained from all participating patients.

Mice

Sucrn $1^{-1-}$ mice or WT mice (9-12 weeks old, 20-25 g weight, kindly provided by Dr Kenneth Mc Creath and Dr Ana Cervera ${ }^{31}$ ) were bred with a C57BI/6 background. All animals were maintained under-specific pathogen-free conditions in the animal facility of the University of Valencia and were co-housed to minimize potential effects of different microbiota. All protocols were approved by the institutional animal care and use committees of the University of Valencia, and all experiments were performed in compliance with the European Animal Research Laws (European Communities Council Directives 2010/ 63/EU, 90/219/EEC, Regulation (EC) No. 1946/2003), and Generalitat Valenciana (Artículo 31, Real Decreto 53/2013).

Induction of experimental colitis

TNBS acute colitis was induced by intrarectal administration of $100 \mu \mathrm{l}$ of TNBS ( $3.5 \mathrm{mg}$ per $20 \mathrm{~g}$ mice) dissolved in $40 \%$ ethanol as previously described. ${ }^{23}$ Body weight was monitored daily and mice were killed by cervical dislocation 2 days after TNBS administration.

Induction of intestinal fibrosis

Intestinal fibrosis was induced in vivo using a heterotopic intestinal transplant model as previously described. ${ }^{27}$ Briefly, colon resections from WT and Sucnr $1^{-1-}$ donor mice, were washed with $0.9 \% \mathrm{NaCl}$ to remove the stool and transplanted subcutaneously into the neck of WT recipient mice. Recipient mice were anesthetized with isoflurane and a small area of the back was shaved. Two incisions were made perpendicularly to the body axis, the grafts were implanted into the pockets and the skin was closed with vicryl 5-stiches. After 7 days, recipient mice were killed by neck dislocation and intestinal grafts were obtained. An adjacent segment of the colon from each donor mouse was kept to be used as autologous control tissue (referred to as day 0 or non-transplanted tissue).

Histological studies

Histological analysis was performed in murine colon samples fixed and embedded in paraffin, sectioned $(5 \mu \mathrm{m})$ and stained with hematoxylin. Histological damage was analyzed as previously described. ${ }^{23}$ Sirius Red staining was performed in order to determine the collagen layer in paraffin-embedded tissues, and the staining was examined under both transmission and polarized light.

Immunohistochemical studies

Immunostaining for SUCNR1 (supplementary table S1) was performed in fixed and paraffin-embedded representative sections $(5 \mu \mathrm{M})$ of intestinal surgical resections from damaged mucosa of CD patients or healthy mucosa from colorectal cancer patients, as previously described. ${ }^{32}$

Immunofluorescence

Immunofluorescence for SUCNR1, CD86, CD206, and a-SMA (supplementary table 1) was performed in frozen intestinal human tissue as previously described. ${ }^{33}$ Samples were visualized with by confocal microscopy (Leica TCS SP8, software LASX).

Isolation of peritoneal macrophages and in vitro polarization Peritoneal macrophages were isolated from WT and Sucnr1 $1^{-1-}$ mice and polarized toward an $\mathrm{M} 1$ and $\mathrm{M} 2$ phenotype as previously described. ${ }^{23}$

\section{Fibroblast isolation}

Primary intestinal fibroblasts were obtained from human intestinal resections or from mouse colons. Briefly, the tissue was cut into small pieces and incubated with HBSS-EDTA $30 \mathrm{~min}$ at $37^{\circ} \mathrm{C}$ in order to remove the epithelial cells. After washing with PBS, a digestion of the small explants with collagenase I $(1 \mathrm{mg} / \mathrm{ml})$, hialuronidase $(2 \mathrm{mg} / \mathrm{ml})$ and DNAse $(1 \mu \mathrm{l} / \mathrm{ml})$ was performed during $30 \mathrm{~min}$ at $37^{\circ} \mathrm{C}$. Finally, the small explants were seeded in a Petri dish and maintained under culture with DMEM high glucose (Sigma-Aldrich) supplemented with FCS 20\%, penicilin/ streptomicin $(100 \mu \mathrm{g} / \mathrm{ml})$, gentamycin $(100 \mu \mathrm{g} / \mathrm{ml})$, amphotericin $B$ $(2 \mu \mathrm{g} / \mathrm{ml})$, and ciprofloxacin $(16 \mu \mathrm{g} / \mathrm{ml})$. Intestinal fibroblasts from passages 3 to 8 were used in all experiments and they were treated with several concentrations of succinate $(0.1-5 \mathrm{mM})$ or TGF $\beta(5 \mathrm{ng} / \mathrm{ml})$ for $24 \mathrm{~h}$.

Small interfering (siRNA) transfection

Primary fibroblasts were transfected with 20 pmol of specific SUCNR1 siRNA (Life Technologies) using Lipofectamine-2000 (Invitrogen Life Technologies) according to the manufacturer's instructions. The efficiency of transfection was determined by analyzing the SUCNR1 mRNA expression.

Protein extraction and western blot

Human primary fibroblasts or homogenized intestinal frozen tissues from human or mice were obtained and total proteins were extracted as previously described. ${ }^{34}$ Protein levels were analyzed by western blot using specific antibodies (supplementary Table 1).

RNA extraction and qPCR analysis

RNA extraction from human or mice tissue was performed using Tripure Isolation reagent (Roche) as previously described. ${ }^{35}$ RNA from macrophages or from primary fibroblasts was isolated using the Illustra RNA Spin Mini (GE Healthcare). RT-PCR for all genes analysed (supplementary table 2,3) was performed using the Prime Script RT reagent Kit (Takara Biotechnology) and qPCR with the Prime Script Reagent Kit Perfect Real Time (Takara Biotechnology). Relative gene expression was expressed as follows: change in expression (fold) $=2^{-\Delta(\Delta C T)}$, where $\Delta C T=C T$ (target) $-C T$ (housekeeping), and $\Delta(\Delta C T)=\Delta C T$ (treated) $-\Delta C T$ (control). $\beta$ actin was used as the housekeeping gene.

Measurements of intracellular $\mathrm{Ca}^{2+}$ levels Intracellular $\mathrm{Ca}^{2+}$ levels were determined in fibroblasts cells using the Fluo-4 AM dye $(3 \mu \mathrm{M})$ for $30 \mathrm{~min}$. Pictures were taken with an DMI3000 inverted fluorescence microscope and LASV4.8 software. 
Perchloric acid extraction

The water-soluble metabolites were extracted using perchloric acid extraction. Briefly, frozen tissues were weighed and homogenized in $6 \%$ perchloric acid. The homogenate was centrifuged $(10,000 \mathrm{rpm}, 15 \mathrm{~min})$, and the supernatant was neutralized ( $\mathrm{KOH}$ $3 \mathrm{M})$ to $\mathrm{pH}$ 7. Potassium perchlorate salt was removed by centrifugation (10,000 rpm, $15 \mathrm{~min})$. The supernatant was analysed by proton NMR spectroscopy.

1H NMR Spectroscopy and succinate metabolite concentration The samples of serum from both healthy donors and CD patients and water-soluble metabolites were delivered to the Metabolomics Service of the Faculty of Medicine-UCIM (Central Research Unit) of the University of Valencia, and kept frozen at a temperature of $-80^{\circ} \mathrm{C}$. Twenty-seven microliters of sodium-3'trimethylsilylpropionate-2,2,3,3-d4 (TSP, $48 \mathrm{mM}$ ) in deuterium oxide was mixed with $473 \mu \mathrm{l}$ of serum and placed in a $5 \mathrm{~mm}$ high-resolution NMR tube (Wilmad, Buena, NJ, USA). NMR spectra were acquired using a standard one-dimensional pulse sequence with water suppression in a Bruker Avance DRX 600 spectrometer (Valencia, Spain) operating at a $1 \mathrm{H}$ frequency of $600.13 \mathrm{MHz}$.) as previously described. ${ }^{36}$ All spectra before analysis were processed through phased baseline correction and the spectra were segmented into $0.005 \mathrm{ppm}$ of wide. The spectra were normalized to total aliphatic spectral area $(0.5-4.5 \mathrm{ppm})$ in order to eliminate differences in total metabolite concentration. Metabolite spin systems and resonances were identified by using the standard Chenomx NMR suite version 8.1 (Chenomx Inc., Edmonton, Alberta, Canada), the Human Metabolome Database (http://www.hmdb.ca) and additional 2D homoand multi-nuclear experiments information in line with previous studies. 37,38

\section{Statistical analysis}

The data were expressed as mean \pm S.E.M. and were compared by analysis of variance (one-way ANOVA; in all cases $\mathrm{F}$ achieved $P<0.05$ and there was no significant variance inhomogeneity) with a Newman-Keuls post hoc correction for multiple comparisons or a $t$-test when appropriate (Graph-Pad Software 6.0). A $P$-value $<0.05$ was considered to be statistically significant. For the body weight changes we used two-way ANOVA followed by Newman-keuls multiple comparison test, setting as variables the treatment and the time. Clinical correlation was analyzed in human samples using Spearman's correlation coefficient.

\section{FUNDING}

This work was supported by the Ministerio de Economia, Industria y Competitividad and the European Regional Development fund of the European Union (ERDF) (SAF2016-80072P), CIBERehd (CB06/04/0071), and Generalitat Valenciana [PROMETEOII/2014/035, UGP-14-164, GV/2018/041].

\section{AUTHOR'S CONTRIBUTIONS}

M.D.B., S.C., D.C.M.-C., J.C.-R., C.H., conceived the experiments and analyzed the data. M.D.B., S.C. wrote the manuscript. D.C.M.-C., J.C.-R., P.S., D.O.-M., L.G.-F., conducted the experiments. J.H., R.A., and F.L., provided human $C D$ tissue samples and provided intellectual input. M.H., G.R., and J.V.E. provided intellectual input. All authors reviewed the manuscript.

\section{ADDITIONAL INFORMATION}

The online version of this article (https://doi.org/10.1038/s41385-018-0087-3) contains supplementary material, which is available to authorized users.

Competing interests: The authors declare no competing interests.

\section{REFERENCES}

1. Wynn, T. A. \& Ramalingam, T. R. Mechanisms of fibrosis: therapeutic translation for fibrotic disease. Nat. Med. 18, 1028-1040 (2012).

2. Rieder, F., Zimmermann, E. M., Remzi, F. H. \& Sandborn, W. J. Crohn's disease complicated by strictures: a systematic review. Gut 62, 1072-1084 (2013).

3. Li, C. \& Kuemmerle, J. F. Mechanisms that mediate the development of fibrosis in patients with Crohn's disease. Inflam. Bowel Dis. 20, 1250-1258 (2014).

4. Rieder, F., Fiocchi, C. \& Rogler, G. Mechanisms, management, and treatment of fibrosis in patients with inflammatory bowel diseases. Gastroenterology 152, 340-350 e346 (2017).

5. Mills, E. \& O'neill, L. A. Succinate: a metabolic signal in inflammation. Trends Cell Biol. 24, 313-320 (2014)

6. Corcoran, S. E. \& O'neill, L. A. HIF1alpha and metabolic reprogramming in inflammation. J. Clin. Invest. 126, 3699-3707 (2016).

7. Tannahill, G. M. et al. Succinate is an inflammatory signal that induces IL-1beta through HIF-1alpha. Nature 496, 238-242 (2013).

8. Li, Y. H., Woo, S. H., Choi, D. H. \& Cho, E. H. Succinate causes alpha-SMA production through GPR91 activation in hepatic stellate cells. Biochem. Biophys. Res. Commun. 463, 853-858 (2015).

9. Xie, N. et al. Glycolytic reprogramming in myofibroblast differentiation and lung fibrosis. Am. J. Respir. Crit. Care. Med. 192, 1462-1474 (2015).

10. Vallee, A., Lecarpentier, Y. \& Vallee, J. N. Thermodynamic aspects and reprogramming cellular energy metabolism during the fibrosis process. Int. J. Mol. Sci. 18, 2537 (2017)

11. Gilissen, J., Jouret, F., Pirotte, B. \& Hanson, J. Insight into SUCNR1 (GPR91) structure and function. Pharmacol. Ther. 159, 56-65 (2016).

12. Tan, J. K., Mckenzie, C., Marino, E., Macia, L. \& Mackay, C. R. Metabolite-sensing G protein-coupled receptors-facilitators of diet-related immune regulation. Annu. Rev. Immunol. 35, 371-402 (2017).

13. De Castro Fonseca, M., Aguiar, C. J., Da Rocha Franco, J. A., Gingold, R. N. \& Leite, M. F. GPR91: expanding the frontiers of Krebs cycle intermediates. Cell Commun. Signal. 14, 3 (2016).

14. Rubic, T. et al. Triggering the succinate receptor GPR91 on dendritic cells enhances immunity. Nat. Immunol. 9, 1261-1269 (2008).

15. Littlewood-Evans, A. et al. GPR91 senses extracellular succinate released from inflammatory macrophages and exacerbates rheumatoid arthritis. J. Exp. Med. 213, 1655-1662 (2016).

16. Van Diepen, J. A. et al. SUCNR1-mediated chemotaxis of macrophages aggravates obesity-induced inflammation and diabetes. Diabetologia 60, 1304-1313 (2017).

17. Serena, C. et al. Elevated circulating levels of succinate in human obesity are linked to specific gut microbiota. ISME J 12, 1642-1657 (2018).

18. Le, C. T., Nguyen, G., Park, S. Y., Choi, D. H. \& Cho, E. H. LY2405319, an analog of fibroblast growth factor 21 ameliorates alpha-smooth muscle actin production through inhibition of the succinate-G-protein couple receptor 91 (GPR91) pathway in mice. PLoS ONE 13, e0192146 (2018).

19. Ariza, A. C., Deen, P. M. \& Robben, J. H. The succinate receptor as a novel therapeutic target for oxidative and metabolic stress-related conditions. Front. Endocrinol. 3, 22 (2012).

20. Shiomi, Y. et al. GCMS-based metabolomic study in mice with colitis induced by dextran sulfate sodium. Inflam. Bowel Dis. 17, 2261-2274 (2011).

21. Osaka, T. et al. Meta-analysis of fecal microbiota and metabolites in experimental colitic mice during the inflammatory and healing phases. Nutrients 9, 1329 (2017).

22. Lissner, D. et al. Monocyte and M1 macrophage-induced barrier defect contributes to chronic intestinal inflammation in IBD. Inflam. Bowel Dis. 21, 1297-1305 (2015)

23. Cosin-Roger, J. et al. The activation of Wnt signaling by a STAT6-dependent macrophage phenotype promotes mucosal repair in murine IBD. Mucosal Immunol. 9, 986-998 (2016).

24. Rogler, G. \& Hausmann, M. Factors promoting development of fibrosis in Crohn's disease. Front. Med. 4, 96 (2017).

25. Rieder, F., Kessler, S., Sans, M. \& Fiocchi, C. Animal models of intestinal fibrosis: new tools for the understanding of pathogenesis and therapy of human disease. Am. J. Physiol. Gastrointest. Liver Physiol. 303, G786-G801 (2012).

26. Goffin, L. et al. Anti-MMP-9 antibody: a promising therapeutic strategy for treatment of inflammatory bowel disease complications with fibrosis. Inflam. Bowel Dis. 22, 2041-2057 (2016).

27. Meier, R. et al. Decreased fibrogenesis after treatment with pirfenidone in a newly developed mouse model of intestinal fibrosis. Inflam. Bowel Dis. 22, 569-582 (2016).

28. Salvador, P. et al. CD16+ macrophages mediate fibrosis in inflammatory bowel disease. J. Crohns Colitis 12, 589-599 (2018).

29. Lawrance, I. C. et al. Cellular and molecular mediators of intestinal fibrosis. J. Crohns Colitis 11, 1491-1503 (2017). 
30. Bernard, K. et al. Glutaminolysis is required for transforming growth factor-beta1induced myofibroblast differentiation and activation. J. Biol. Chem. 293, 1218-1228 (2018).

31. Mccreath, K. J. et al. Targeted disruption of the SUCNR1 metabolic receptor leads to dichotomous effects on obesity. Diabetes 64, 1154-1167 (2015).

32. Cosin-Roger, J. et al. M2 macrophages activate WNT signaling pathway in epithelial cells: relevance in ulcerative colitis. PLOS ONE 8, e78128 (2013).

33. Ortiz-Masia, D. et al. Hypoxic macrophages impair autophagy in epithelial cells through Wnt1: relevance in IBD. Mucosal Immunol. 7, 929-938 (2014).

34. Macias-Ceja, D. C. et al. Stimulation of autophagy prevents intestinal mucosal inflammation and ameliorates murine colitis. Br. J. Pharmacol. 174, 2501-2511 (2017).
35. Ortiz-Masia, D. et al. Induction of CD36 and thrombospondin-1 in macrophages by hypoxia-inducible factor 1 and its relevance in the inflammatory process. PLoS One 7, e48535 (2012).

36. Monleon, D. et al. Metabolite profiling of fecal water extracts from human colorectal cancer. NMR Biomed. 22, 342-348 (2009).

37. Bodi, V. et al. Metabolomic profile of human myocardial ischemia by nuclear magnetic resonance spectroscopy of peripheral blood serum: a translational study based on transient coronary occlusion models. J. Am. Coll. Cardiol. 59, 1629-1641 (2012).

38. Garcia-Simon, M. et al. Prognosis biomarkers of severe sepsis and septic shock by $1 \mathrm{H}$ NMR urine metabolomics in the intensive care unit. PLOS ONE 10, e0140993 (2015). 